Tatiana I. Becker*, Yuriy L. Raikher, Oleg V. Stolbov, Valter Böhm and Klaus Zimmermann

\title{
Magnetoactive elastomers for magnetically tunable vibrating sensor systems
}

\begin{abstract}
Magnetoactive elastomers (MAEs) are a special type of smart materials consisting of an elastic matrix with embedded microsized particles that are made of ferromagnetic materials with high or low coercivity. Due to their composition, such elastomers possess unique magnetic field-dependent material properties. The present paper compiles the results of investigations on MAEs towards an approach of their potential application as vibrating sensor elements with adaptable sensitivity. Starting with the model-based and experimental studies of the free vibrational behavior displayed by cantilevers made of MAEs, it is shown that the first bending eigenfrequency of the cantilevers depends strongly on the strength of an applied uniform magnetic field. The investigations of the forced vibration response of MAE beams subjected to inplane kinematic excitation confirm the possibility of active magnetic control of the amplitude-frequency characteristics. With change of the uniform field strength, the MAE beam reveals different steady-state responses for the same excitation, and the resonance may occur at various ranges of the excitation frequency. Nonlinear dependencies of the amplification ratio on the excitation frequency are obtained for different magnitudes of the applied field. Furthermore, it is shown that the steady-state vibrations of MAE beams can be detected based on the magnetic field distortion. The field difference, which is measured simultaneously on the sides of a vibrating MAE beam, provides a signal with the same frequency as the excitation and an amplitude proportional to the amplitude of resulting vibrations. The presented prototype of the MAE-based vibrating unit with the field-controlled "configuration" can be implemented for realization of acceleration sensor systems with adaptable sensitivity. The ongoing research on MAEs is oriented to the use of other geometrical forms along with beams, e.g. two-dimensional structures such as membranes.
\end{abstract}

\footnotetext{
*Corresponding author: Tatiana I. Becker, Technical Mechanics Group, Faculty of Mechanical Engineering, Technische Universität Ilmenau, Ilmenau, Germany, E-mail: tatiana.becker@tuilmenau.de

Yuriy L. Raikher and Oleg V. Stolbov, Laboratory of Physics and Mechanics of Soft Matter, Institute of Continuous Media Mechanics, Ural Branch of Russian Academy of Sciences, Perm, Russia,

E-mail: yuriy.raikher@gmail.com (Y.L. Raikher), oleg100@gmail.com (0.V. Stolbov)

Valter Böhm, Faculty of Mechanical Engineering, Ostbayerische Technische Hochschule Regensburg, Regensburg, Germany, E-mail: valter.boehm@oth-regensburg.de

Klaus Zimmermann, Technical Mechanics Group, Faculty of Mechanical Engineering, Technische Universität Ilmenau, Ilmenau, Germany, E-mail: klaus.zimmermann@tu-ilmenau.de
}

Ә Open Access. ( 2020 Tatiana I. Becker et al., published by De Gruyter. (c) BY-NC-ND This work is licensed under the Creative Commons Attribution-NonCommercial-NoDerivatives 4.0 International License.

https://doi.org/10.1515/psr-2019-0125 
Keywords: adaptable sensor unit, amplification ratio, bending vibration, eigenfrequency, magnetoactive elastomer, magnetic field control

\section{Introduction}

Magnetoactive elastomers (MAEs) consist of magnetically soft and/or magnetically hard particles of microsize that are embedded into a nonmagnetic elastic matrix (Figure 1). This class of smart materials has potentially an extending field of application in diverse technical areas. Just like magnetorheological fluids and ferrofluids [1, 2], MAEs strongly respond to magnetic fields. Experimental investigations on MAEs with magnetically soft particles show that their mechanical properties can be changed sufficiently, when they are subjected to an external magnetic field, e.g. [3, 4]. It is found that the elastic modulus of these materials is "tunable", which means its effective increase in the presence of an applied magnetic field [5-7]. The reasons for this active effect of "tuning" are interactions between the different types of magnetic filler and the matrix in a microscopic scale and the subsequent structure formation of the particles.

Recent studies on MAEs with embedded magnetically hard particles show that they possess striking properties, particularly remanent magnetization, even in the absence of an external field [8-10]. Once such freshly synthesized elastomer when subjected to a strong magnetic field acquires a remanent magnetization and becomes actually an elastic magnet with modified properties as compared to the original elastomer. This effect of passive magnetic control of properties depends on ferromagnetic properties and concentration of magnetically hard particles as well as the field magnitude applied during the initial magnetization [11]. A special type of MAEs with a mixed magnetic filler made of magnetically hard and soft particles is of particular research interest at present. Such elastomers with a mixed magnetic filler show not just pure passive and active effects that elastomers containing only one type of particles would have [12-14]. The interplay of two fillers different in their ferromagnetic properties results in a complex distribution of the magnetic field, which in turn influences the field reaction of the material itself.

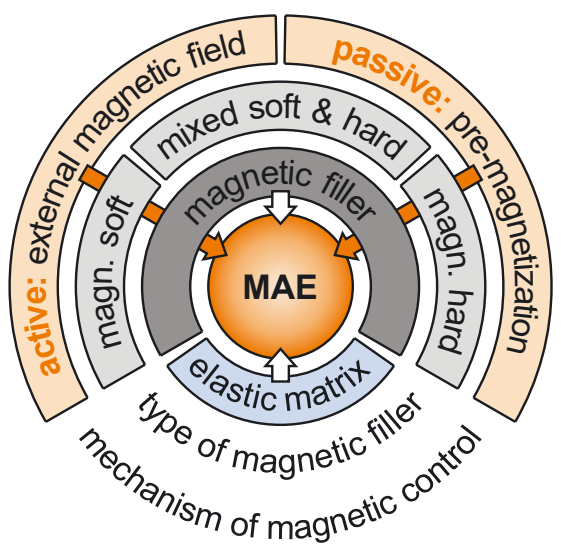

Figure 1: MAE material systems with different magnetic fillers. 
Magnetic and viscoelastic properties of these materials, depending on magnetic field, are studied using various experimental approaches in [6,14-16]. Specific features of MAEs can be also identified by the magnetization analysis based on first-order reversal curve measurements $[17,18]$. To shed light on the particle characterization and structuring, the imaging technique such as X-ray microtomography is used [19]. The extracted data of the position, rotation and size of individual particles gives an input for theoretical modeling and simulation studies of MAEs. From a methodological point of view, these investigations follow two main modeling approaches: dipole-interaction models [20-23] and finite element methods based on a continuum formulation [24, 25]. Mesoscopic scale models, that allow to predict shape deformations of MAEs with magnetically hard and soft particles in a magnetic field, are developed in [26, 27].

Due to their exceptional field-dependent properties, smart materials like MAEs offer great potential for designing intelligent technical applications. The fact that a magnetic field might be exerted contactlessly makes them unique for a number of applications in the area of vibration isolation and damping [28, 29]. A variable differential mount apparatus using an MAE for improving driving comfort of a vehicle is presented in [30]. In the field of biomechanics, prosthetic and orthotic devices with shock absorption springs made of magnetic elastomers can be adjusted to a level corresponding to an activity of the user [31]. Magnetorheological fluids in terms of their application in medical and rehabilitation devices are discussed in [32, 33]. By using the influence of a magnetic field on the mechanical deformation of MAEs, various actuation and locomotion systems can be developed [34, 35]. A method to program the magnetic actuation in order to achieve complex time-varying shapes of MAEs is proposed in [36]. This may lead to a vast number of miniature soft devices mimicking biological beating patterns, e.g. an artificial cilium. A nature-inspired flexible gripper made of an MAE with the remote actuation is shown in [37]. Another example is a soft robotic form-fit gripper based on the field-induced plasticity effect of MAEs [38, 39]. This gripper is able to adapt its shape to the geometry of gripped objects, and the shape remains preserved after a mechanical load when a large magnetic field is present. As soon as the magnetic field is removed, the original shape of the MAE gripper is restored. Various film structures of anisotropic MAEs with a directional field response can be used for applications in soft robotics [40].

An object of this study is to assess the potential of MAEs to design a sensor system with an adjustable sensitivity controlled by a magnetic field. Following our previous work [41], we study an oscillatory motion of MAE macro beams that might serve as functional elements for fabricating sensors of several kinds, e.g., acceleration, force and pressure sensors. The concept to design a system incorporating a sensor element made of MAE to detect mechanical stimuli of the environment, is presented in [7]. According to it, an MAE beam structure with a mixture of magnetically soft and hard particles shows bending vibrations induced by an external excitation of its base. This response can be identified by measurements of the magnetic field distortion using Hall sensors that are spatially distributed around the vibrating element. The resulting 
signals then could be converted in a facile way into electromagnetic ones for measuring and analyzing. The key point is that an adaptivity of the MAE sensor element can be realized by means of an externally applied magnetic field, which leads to the change of the material properties, above all the mechanical compliance, due to the complex particle-matrix interactions. This means that the sensor element displays different behavior without and with an external field, and its "sensitivity" can be adjusted depending on the given field magnitude. Note that the proposed sensor differs from other oscillatory schemes employing MAEs, see [42, 43], for example, by using a uniform, no gradient magnetic field whose magnitude, therefore, does not depend on the position of the MAE. In other words, the field works as a tunable bias factor defining the conditions under which the sensor operates [41].

In essence, the most comprehensible working process of the scheme [7] is the camertone-type oscillation of the MAE cantilever, which in general has a wide set of eigenfrequencies. However, if the beam is sufficiently massive, its basic (the lowest) eigenfrequency is quite far even from the first overtone. This can facilitate the filtering of the signal and the analysis of the system under monitoring. Hence, the proposed concept requires detailed investigations of free bending vibrations of MAEs. Section 2 shows the experimental results on the vibration characteristics of MAE beams of different geometry and magnetic filling dependent on an applied, almost homogeneous magnetic field. It also includes the theoretical analysis based on the developed magneto-mechanical model for determination of eigenfrequencies of MAE beams. Section 3 presents the prototype of an MAE-based vibrating unit and results on its forced bending vibrations without and with an external uniform magnetic field. The detection of the MAE vibrations using methods of the magnetic field sensing are discussed in Section 4. Finally, conclusions are outlined in Section 5.

\section{Magnetic field-dependent vibration characteristics of MAEs}

Considering the dynamic behavior of functional sensor elements made of MAEs [38], one of the major issues is the dependence of the material properties on an externally applied magnetic field that primarily influences vibration characteristics. The design of MAE vibration elements involves the determination of both geometry and material properties. Dimensions of an MAE beam and its material composition (i.e., elastic matrix material, concentration and type of the magnetic filler) are to be chosen as to make the first eigenfrequency be in a specific range assigned in advance corresponding to the controlling magnetic field. In what follows, we present experimental and theoretical methods of determination of the first eigenfrequency of MAE beams, taking into account magneto-mechanical interactions [38]. 


\subsection{Experimental investigation of free bending vibrations of MAE cantilevers in a uniform magnetic field}

To get a qualitative notion of the problem, consider an MAE beam that hangs vertically being fixed by its upper end and is excited by imposing a small horizontal displacement on the lower free end [41]. During the bending vibrations of the beam on account of its continuity, the displacement amplitude is maximal at the free end and falls gradually down to zero at the fixed end. The same relates to the rotation angle between the tangent to the neutral axis of the beam and the vertical. Knowing the eigenfrequencies and corresponding modes of vibrations of the MAE beam, one can predict the emergence of resonance, and therefore permit or avoid it by relevant variation of an applied magnetic field.

\subsubsection{Materials and methods}

The oligomer base for the MAEs used in tests is silicone Alpa-Sil Classic (Alpina Co.) with Shore-A hardness 6-8, in which the magnetically soft particles of carbonyl iron powder (CIP)-CC (BASF Co.) with an averaged diameter of $6 \mu \mathrm{m}$ are mechanically stirred up. Due to the high viscosity of the paste-like mixture and the short polymerization time, isotropic beam samples with a homogeneous distribution of the magnetic filler are produced. Four different volume concentrations of the CIP are used: $\phi=0,0.1,0.2$ and 0.3. The material parameters are listed in Table 1.

The geometry of an MAE beam is shown in Figure 2. It has rounded edges, so as to smooth the magnetic field distortions at the corners. The cross-section of the beam is

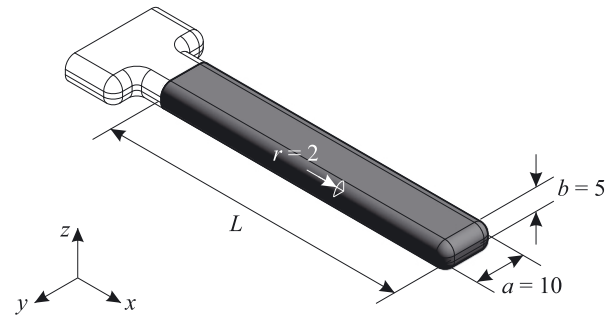

Figure 2: Geometry of the MAE beam, dimensions are given in $\mathrm{mm}$.

Table 1: Material parameters of MAE beam samples.

\begin{tabular}{lrrrr}
\hline Composition No. & $\mathbf{1}$ & $\mathbf{2}$ & $\mathbf{3}$ & $\mathbf{4}$ \\
\hline CIP volume concentration, $\phi$ & 0 & 0.1 & 0.2 & 0.3 \\
Mass density, $\rho\left(10^{3} \mathrm{~kg} \mathrm{~m}^{-3}\right)$ & 1.06 & 1.74 & 2.42 & 3.10 \\
\hline
\end{tabular}




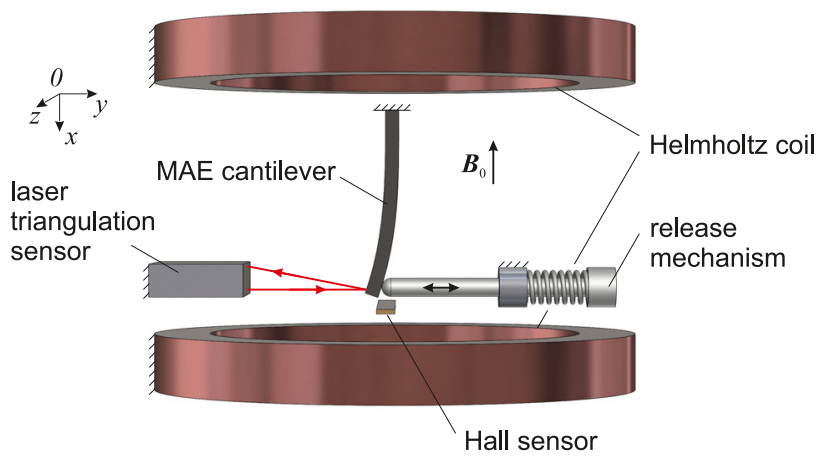

Figure 3: Experimental setup used for the investigation of free bending vibrations of an MAE cantilever in the presence of a uniform magnetic field. Reprinted by permission from [41].

nearly a rectangle of $a \times b=10 \mathrm{~mm} \times 5 \mathrm{~mm}$ with a corner radius of $r=2 \mathrm{~mm}$. The cross-sectional area is $A=46.57 \mathrm{~mm}^{2}$. Its area moment of inertia equals to $I_{z}=89.21 \mathrm{~mm}^{4}$. Samples of three different beam lengths are prepared: $L=40,60$ and $80 \mathrm{~mm}$. The "transverse" part of the T-form is designed for fixing the sample inside the support. Altogether, $12 \mathrm{MAE}$ beam samples of three different lengths and four different compositions are used in the experimental studies.

The setup shown in Figure 3 is used to investigate the vibration behavior of an MAE beam in the presence of an external magnetic field. It consists of a pair of Helmholtz coils placed symmetrically along the vertical $x$ axis. The magnetic flux density $B_{0}$ induced by the coils in the relevant region between them is up to $60 \mathrm{mT}$ and points straight vertically. This is proven experimentally by measurements in air with a Hall sensor and also simulated using finite element method. Both tests render the magnetic field homogeneity not worse than $96.5 \%$ [41].

An MAE beam is held by its top end in a fixed nonmagnetic support and positioned in the middle between the Helmholtz coils (Figure 3). Such construction makes, therefore, a kind of cantilever. In equilibrium, the central axis of the beam is co-aligned with the symmetry axis of the coil. The initial deflection of the beam in the $x$ - $y$ plane is created using a mechanical release mechanism. It consists of a threaded rod which is screwed against the pressure spring to adjust an initial deflection of the cantilever's free end of $5 \mathrm{~mm}$ along the $y$ axis. When the trigger is pulled, the tensioned spring snaps the rod back, and the cantilever is released.

Time-dependent displacement of the cantilever's free end is measured using a laser triangulation sensor. The preliminary investigations show that due to the symmetry of the beam, the bending vibrations take place principally in the $x-y$ plane of the initial deflection. Additionally, the distortion of the applied magnetic field close to the free end of the sample can be measured by a Hall sensor [41]. 


\subsubsection{Results of free bending vibrations of MAE cantilevers}

Experimental examination of the free bending vibrations of the MAE cantilevers in the vertical $x-y$ plane is performed for various magnitudes of an applied uniform magnetic field from zero up to $60 \mathrm{mT}$ in steps of $10 \mathrm{mT}$. For each beam sample and each field magnitude, the vibration behavior of the beam is analyzed based on 12 repetitions for both orientations in the fixed support.

The results indicate that, in contrast to the cantilevers made of pure silicone, the oscillatory dynamics of each sample comprising CIP particles can be changed essentially by means of the uniform magnetic field. Figure 4 illustrates the displacement measurements for the MAE cantilever with a CIP volume concentration of $\phi=0.3$ and length of $L=60 \mathrm{~mm}$ for the two extreme values of $B_{0}$. As it could be seen, the vibrations are lightly damped, and their period is with a good accuracy isochronous, i.e., it is independent of the amplitude. This enables us to treat the vibrating system under study as a linear one.

From the data underlying Figure 4 (b), it follows that in the absence of the field the period of vibrations is $0.245 \mathrm{~s}$, and the first eigenfrequency is $f_{1}(0)=4.08 \mathrm{~Hz}$. For $B_{0}=60 \mathrm{mT}$, we find $f_{1}\left(B_{0}\right)=6.74 \mathrm{~Hz}$. Thus, for this MAE beam, the maximum relative change of the first eigenfrequency is $R_{f_{1}}=f_{1}\left(B_{0}\right) / f_{1}(0)-1=65.21 \%$. This relative change of the eigenfrequency can be related to the field-induced effective increase of the Young modulus $E$ of the MAE material from $E(0)=0.69 \mathrm{MPa}$ to $E_{\text {eff }}\left(B_{0}\right)=2.36 \mathrm{MPa}$. Note that the observed tunability of the vibration characteristics is reversible when a moderate magnetic field of $60 \mathrm{mT}$ is removed. For strong fields, irreversible effects of the magnetic particle movement in the elastic matrix may take place.

Using the logarithmic decrement, which is the ratio of any two successive amplitudes, the dimensionless damping ratio $D$ is obtained. For all cantilevers used in tests, $D<0.05$ that corresponds to the underdamped case, and it decreases depending on the field magnitude. Just as for a system with finite number of degrees of freedom, it may be

(a)

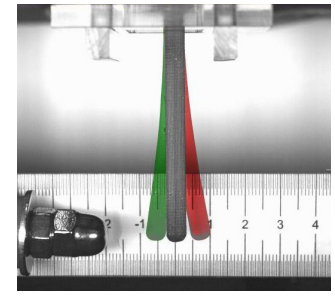

(b)

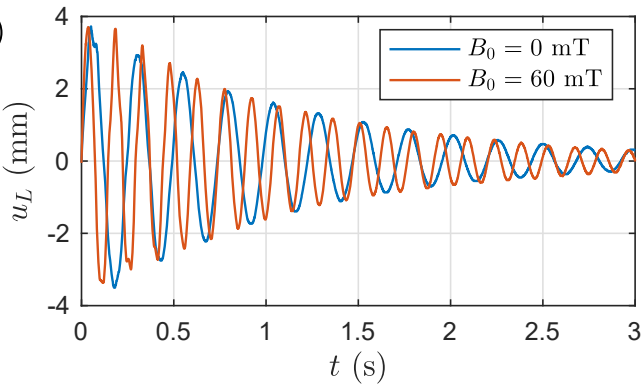

Figure 4: Free bending vibrations of the MAE cantilever of $L=60 \mathrm{~mm}$ and $\phi=0.3$ : (a) Overlapped sequential frames of the video recorded at $750 \mathrm{fps}$ without magnetic field; (b) time-dependent displacement $u_{L}(t)$ of the free end for $B_{0}=0$ and $60 \mathrm{mT}$. Reprinted by permission from [41]. 

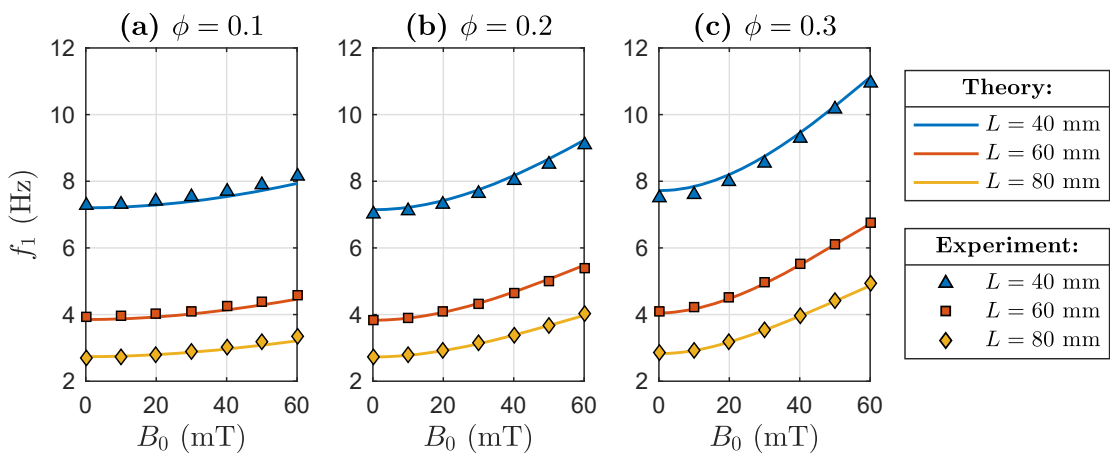

Experiment:

$\Delta L=40 \mathrm{~mm}$

ㅁ $L=60 \mathrm{~mm}$

$\diamond \quad L=80 \mathrm{~mm}$

Figure 5: The first eigenfrequency $f_{1}$ of the free bending vibrations depending on the magnetic field magnitude $B_{0}$ for MAE cantilevers of different lengths $L$ with CIP volume concentrations: (a) $\phi=0.1$; (b) $\phi=0.2$; (c) $\phi=0.3$. Experimental values (markers) represent the mean values of 12 measurements. Theoretical dependencies (solid lines) are obtained for parameters $X_{\mathrm{e}}=79$ and $k_{\mathrm{m}}=0.72$. Reprinted by permission from [38].

assumed that the damping ratio $D=\pi \beta_{1} f_{1}$ is proportional to the internal damping coefficient $\beta_{1}$ during harmonic vibrations at the eigenfrequency $f_{1}$.

The first eigenfrequency $f_{1}\left(B_{0}\right)$ of the free bending vibrations obtained from the displacement measurements for all MAE cantilevers is shown in Figure 5. These dependencies increase monotonically with the magnitude of the uniform magnetic field. The maximum relative change $R_{f 1}=73.56 \%$ is observed for the longest sample of $L=80 \mathrm{~mm}$ and with a maximum CIP concentration of $\phi=0.3$. Thus, the vibration characteristics of the MAE beams and, above all, their mechanical compliance can be controlled and reversibly tuned by means of an external uniform magnetic field.

\subsection{Magneto-mechanical modeling for determination of MAE eigenfrequencies}

The elastic forces and torques acting on every beam element are induced due to a combination of elastic, inertial and gravity forces. This case is well known, and the pertinent equations of motion could be taken from the literature $[44,45]$. For a cantilever made of an MAE, one may now modify the behavior of the system by subjecting it to a uniform magnetic field along the vertical (up or down) direction. This transforms the mechanical problem into a magneto-mechanical one, where it is needed to consider, among usual forces and torques, the one that is caused by the ponderomotive (magnetic) force [41].

\subsubsection{Model expression for the magnetic torque}

Due to magnetostatic reasons, the MAE cantilever when positioned straight and parallel to the applied field has the configuration of the lowest magnetic energy. Any 


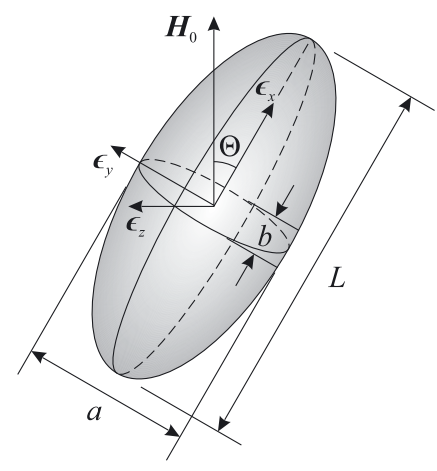

Figure 6: MAE ellipsoid in a uniform magnetic field used to derive the model expression of the magnetic torque. Reprinted by permission from [38].

distortions of this shape increase the magnetic energy, and thus induces ponderomotive forces striving to restore the configuration. Evidently, these forces affect the cantilever's eigenfrequency, the greater the stronger the magnetic field. In other words, by measuring the frequency change of the bending vibrations, one is able to extract information on the field strength, and vice versa. By knowing the magnitude of the applied uniform field, it is possible, based on the extended magneto-mechanical model presented below, to obtain theoretically the eigenfrequencies of MAE vibrating units [41].

In such a sample, the magnetization depends not only on the magnetic susceptibility of an MAE material, but also on the shape of the beam. The magnetic poles appearing on its ends produce a demagnetizing field, which is proportional and directed opposite to the magnetization. For the prismatic MAE beam used in the experiment (Figure 2), the magnetic field is not uniform and varies from place to place in the beam, being maximum in the middle. In order to calculate analytically the field inside a prismatic MAE beam, the approximation of its shape by a magnetizable general ellipsoid with the same dimensions $L>a>b$ of the principle axes is used (Figure 6). The magnetic susceptibility $\chi$ of the MAE is assumed to be constant and independent of the magnetic field and strain. Those simplifications are justified by the fact that a lowto-moderate magnetic field range $(<100 \mathrm{mT})$ and small deformations of the MAE beam are considered [38].

Assume a uniform external magnetic field $\boldsymbol{H}_{0}=H_{0}\left(\cos \Theta \epsilon_{x}+\sin \Theta \epsilon_{y}\right)$ applied along the vertical axis, where $\epsilon_{\boldsymbol{x}, \boldsymbol{y}, \boldsymbol{z}}$ are the unit vectors directed along the ellipsoid principle axes, and $\boldsymbol{\Theta}$ is the angle between $\epsilon_{\boldsymbol{x}}$ and the vertical (Figure 6). Then, the magnetic field $\boldsymbol{H}$ inside the ellipsoid is shown to be uniform and defined by $[41,46]$ :

$$
\boldsymbol{H}=H_{0}\left(\frac{\cos \Theta}{1+\chi N_{x x}} \epsilon_{x}+\frac{\sin \Theta}{1+\chi N_{y y}} \epsilon_{y}\right),
$$


where $N_{x x}$ and $N_{y y}$ are components of the diagonalized demagnetization tensor that depend only on the aspect ratios $a / L$ and $b / L$ of the ellipsoid [47].

The magnetic susceptibility $\chi$ in (1) should be taken as an effective value $\chi_{\mathrm{e}}$ rendering that of the MAE with the given volume concentration $\phi$ of the CIP filler. This dependence can be approximated by the Lichtenecker formula [48], which is quite reliable for the composites with $\phi$ up to 0.3 . Starting from its conventional expression for the magnetic permeability $\mu(\phi)=\mu_{\mathrm{e}}^{\phi}$, one can obtain an expression in terms of susceptibility:

$$
\chi(\phi)=\left(\chi_{\mathrm{e}}+1\right)^{\phi}-1 .
$$

For the linear magnetization law, the magnetic moment of the ellipsoid is defined as $\boldsymbol{m}=\chi(\phi) V_{\mathrm{el}} \boldsymbol{H}$ with the ellipsoid volume $V_{\mathrm{el}}=\pi a b L / 6$.

The magnetic energy of the ellipsoid tilted under the angle $\Theta$ to the direction of the field is $U_{\text {mag }}=-\frac{1}{2} \mu_{0} \boldsymbol{m} \boldsymbol{H}_{0}$, where $\mu_{0}$ is vacuum magnetic permeability. As seen from (1), the direction of the magnetic moment $m$ does not coincide with that of the applied field $H_{0}$, due to which the ellipsoid experiences a field-induced torque $T=T \epsilon_{z}$ along the $z$ axis. Its magnitude $T$ is determined by the first derivative of the magnetic energy with respect to the rotation angle. Assuming small angular deviations $\Theta$ « 1 , the following expression for the ponderomotive torque can be obtained [38]:

$$
T=-\frac{\partial U_{\mathrm{mag}}}{\partial \Theta}=\mu_{0} H_{0}^{2} V_{\mathrm{el}} \Theta W\left(\chi_{\mathrm{e}}, \frac{a}{L}, \frac{b}{L}\right)
$$

where the notation $W$ called the form-factor is introduced as:

$$
W\left(\chi_{\mathrm{e}}, \frac{a}{L}, \frac{b}{L}\right)=\frac{\chi^{2}(\phi)\left(N_{x x}-N_{y y}\right)}{\left(1+\chi(\phi) N_{x x}\right)\left(1+\chi(\phi) N_{y y}\right)} .
$$

This means that under weak perturbations the ponderomotive torque $T$ acting on the ellipsoid is quadratic in the applied field strength and linear in the rotation angle, while the form-factor $W$ takes into account the magnetic properties and the anisotropy of the beam. Note that the smallest component of the demagnetization tensor always corresponds to the longest dimension of the body. Therefore, in (4) the difference $N_{x x}-N_{y y}$ is negative that ensures that the torque $T$ is restoring.

\subsubsection{Equations of motion of vibrating MAE cantilever}

To evaluate the eigenfrequencies of an elongated MAE cantilever, the thin-rod approximation is considered [44]. A uniform magnetic field $H_{0}$ is directed vertically, i.e., is coaligned with the gravity field $g$. Under these conditions, it is evident that in 
equilibrium the cantilever dwells in a straight vertical configuration, and its fieldinduced magnetic moment $m$ is parallel to the applied field.

During the bending vibrations of the MAE cantilever in the vertical $x-y$ plane, there are three factors that generate restoring torques striving to recover the initial equilibrium configuration: the elasticity of the material, gravity and ponderomotive (magnetostatic) torque. All of them are included in the dynamic equations of motion, in which the rotary inertia is accounted for, while the viscous forces (dissipation) are neglected. In other words, keeping in mind the experimental evidence shown in Figure 4, we assume that the beam restores its equilibrium configuration by way of lightly damped small vibrations [41].

The set of equations of motion for the in-plane small free bending vibrations is as follows [38]:

$$
\begin{aligned}
\rho A \frac{\partial^{2} u(x, t)}{\partial t^{2}}= & \frac{\partial Q_{y}(x, t)}{\partial x}, \\
\rho I_{z} \frac{\partial^{2} \vartheta(x, t)}{\partial t^{2}}= & \frac{\partial M_{z}(x, t)}{\partial x}+Q_{y}(x, t)+\rho g A(x-L) \vartheta(x, t) \\
& +k_{m} \mu_{0} A H_{0}^{2} W\left(\chi_{e}, \frac{a}{L}, \frac{b}{L}\right) \vartheta(x, t), \\
\frac{\partial \vartheta(x, t)}{\partial x}= & \frac{M_{z}(x, t)}{E I_{z}}, \quad \frac{\partial u(x, t)}{\partial x}=\vartheta(x, t) .
\end{aligned}
$$

Here, the coordinate $x$ varies from 0 to $L, t$ is the time, $u(x, t)$ is the horizontal displacement of the beam points in the $O y$ direction, $\vartheta(x, t)$ is the rotation angle around the $z$ axis, $Q_{y}(x, t)$ is the component of the shear force, $M_{z}(x, t)$ is the bending moment in the $O z$ direction. The other parameters entering (2.2.2) are: $\rho$ the mass density of the MAE, $A$ the cross-sectional area of the beam, $I_{z}$ the area moment of inertia, and $E$ the Young modulus of the MAE.

To compensate the approximation discrepancy between the geometry of an MAE prismatic beam and ellipsoid, an adjustable parameter $k_{\mathrm{m}}$, appearing as a multiplier to the term of the ponderomotive torque, is introduced in the second equation of (5). The other parameter $\chi_{\mathrm{e}}$, namely the effective magnetic susceptibility of CIP, enters the set of equations in a nonlinear way, as is seen from (2) and (4).

This model is applied for the determination of eigenfrequencies of the MAE cantilevers by considering the fixed-free boundary conditions: no deflection and rotation at $x=0$, and no bending moment and shear force at $x=L$.

The numerical method of initial parameters is used [44, 41]. The calculations are performed for the material and geometrical parameters corresponding to the MAE cantilevers used in the experiment, see Section 2.1.1. For the parameters $\chi_{\mathrm{e}}$ and $k_{\mathrm{m}}$ entering the theoretical model, different sets of values in the ranges $k_{\mathrm{m}}<1$ and 
$40<\chi_{\mathrm{e}}<140$ are examined. The latter is the range of magnetic susceptibility measured for compacted CIP [49].

\subsection{Comparison of experimental and theoretical results of the first eigenfrequency}

According to [41], the proposed model is applied for interpretation of the experimental data. As a first step, by fitting the experimental eigenfrequencies $f_{1}(0)$ of the MAE cantilevers measured in zero magnetic field (Figure 5), the Young moduli $E$ of the MAE compositions with different CIP volume concentrations $\phi$ are evaluated (Table 2).

Then the first eigenfrequencies of the MAE cantilevers are calculated numerically for different magnitudes of the applied magnetic field using the corresponding averaged values of the Young modulus $E(\phi)$. For each particular sample of length $L$ and concentration $\phi$, the parameters $\chi_{\mathrm{e}}$ and $k_{\mathrm{m}}$ entering the theoretical model are obtained from fitting the experimental frequency data. It turned out from calculations that values $\chi_{\mathrm{e}}=79$ and $k_{\mathrm{m}}=0.72$ are "universally" valid, i.e., they come out virtually whatever sample is chosen for fitting. Comparison between experimental and numerical data on the first eigenfrequency as a function of the cantilever length is given in Figure 5. It can be seen that the numeric solution with the above-given particular values of $\chi_{\mathrm{e}}$ and $k_{\mathrm{m}}$ fairly well (percent error below 5.6\%) describe the measurement results [41].

The essence of the undertaken modification is the inclusion of an expression for the ponderomotive torque, which is exerted by the applied uniform magnetic field on the magnetized MAE cantilever, to the dynamical equations of thin rods. The fitting procedure yields the value of $k_{\mathrm{m}}=0.72$ below unity. At the first sight, this implies that the effective magnetic torque density is somewhat smaller than that generated by the model ellipsoid. Note, however, that the magnetic term in (5) is the product $k_{\mathrm{m}} W$, where the form-factor $W$ is given by (4). Although $W$ is a nonlinear function of the magnetic

Table 2: Young moduli of the MAE cantilevers of different length $L$ [41].

\begin{tabular}{lcccr}
\hline Composition No. & $\mathbf{1}$ & $\mathbf{2}$ & $\mathbf{3}$ & $\mathbf{4}$ \\
\hline CIP volume concentration, $\phi$ & 0 & 0.1 & 0.2 & 0.3 \\
Young modulus, $E\left(10^{5} \mathrm{~Pa}\right):$ & & & 4.12 & 6.17 \\
$L=40 \mathrm{~mm}$ & 2.09 & 3.24 & 4.34 & 6.84 \\
$L=60 \mathrm{~mm}$ & 2.08 & 3.37 & 4.41 & 6.88 \\
$L=80 \mathrm{~mm}$ & 2.22 & 2.83 & 4.29 & 6.63 \\
Averaged young modulus, $E(\phi)\left(10^{5} \mathrm{~Pa}\right)$ & 2.13 & 3.14 & & \\
\hline
\end{tabular}


susceptibility, the leading contribution of the product is $k_{\mathrm{m}} \chi^{2}(\phi)$. Therefore, it is mostly the value of this product that matters for the adjustment, so that greater $k_{\mathrm{m}}$ means smaller the effective susceptibility $\chi_{\mathrm{e}}$. As to the latter, the used value $\chi_{\mathrm{e}}=79$ is rendered by a more precise fitting procedure that takes into account the full nonlinear dependence $W\left(\chi_{\mathrm{e}}\right)$. This value looks quite reasonable if it is to be compared with the data from [49], which puts for measured permeability values of compacted CIP in the range from 40 to 140 .

From symmetry considerations, it follows that the dependence of $f_{1}\left(B_{0}\right)$ for a weak applied field should be quadratic. Qualitatively, this can be seen from the initial parts of the curves in Figure 5. A fairly good agreement obtained demonstrates the reliability of the developed magneto-mechanical model for determination of the eigenfrequencies of an MAE cantilever.

\section{Forced vibration response of MAE in a uniform magnetic field}

The possibility to change in a great extend the vibration characteristics of MAE beams by means of an active magnetic control can be potentially implemented for realization of sensor systems with adjustable sensitivity. A prototype of an MAE-based vibrating unit for detection of external mechanical stimuli is presented in Figure 7. Its main functional element is a double-fixed MAE beam placed in the inner region of the Helmholtz coil. In this section, the focus is on the experimental investigation of forced bending vibrations displayed by the MAE under the influence of a uniform magnetic field. This will pave the way to adjust the vibration response of such unit, and thus to realize its adaptable sensitivity. In addition, a theoretical approach using an oscillator model with a cubic stiffness term is considered in order to describe the resulting nonlinear behavior of the MAE beam.

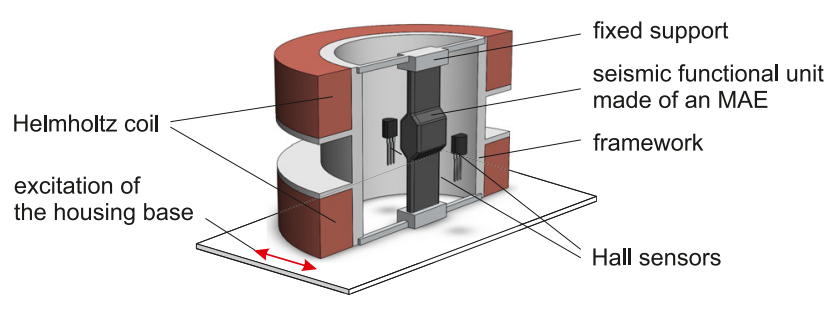

Figure 7: Prototype of an MAE-based vibrating sensor element with magnetic field-adjustable vibration characteristics for detection of external mechanical stimuli. Reprinted by permission from [38]. 


\subsection{Experimental investigation of forced bending vibrations of the MAE unit}

\subsubsection{Experimental setup and methods}

The setup used to investigate the forced bending vibrations of a double-fixed MAE beam in the presence of an external magnetic field is shown schematically in Figure 8. The MAE beam is fastened vertically at both ends to a housing. Its kinematic excitation is realized in the following way. The housing is attached by a rod to an electrodynamic vibration test system. It is controlled by a waveform generator, whose sinusoidal signal is strengthened using a power amplifier. The core of the vibration test system moves in the manner described by the harmonic function $u_{0}(t)=u_{0} \cos (2 \pi \Omega t)$ in the horizontal direction along the rod's axis, i.e., perpendicular to the beam's axis in equilibrium. The excitation amplitude $u_{0}$ is specified to be in a narrow range of $0.4 \pm 0.02 \mathrm{~mm}$. The excitation frequency $\Omega$ is set to a value from the range of $5 \mathrm{~Hz}$ up to $22 \mathrm{~Hz}$. For each measurement, the frequency is held constant, and then it is changed gradually only in one direction (either upwards or downwards) for subsequent measurements. To record large responses near resonance, the minimum step size for the frequency setting is $0.01 \mathrm{~Hz}$. To detect resulting in-plane bending vibrations of the MAE beam, the laser triangulation sensor is used. Its output signal is transmitted to a digital oscilloscope and analyzed using a PC software [50].

The magnetic field is produced by a Helmholtz coil, consisting of two parallelmounted circular coils that have a radius of $56.5 \mathrm{~mm}$ and a winding number of 855 . The cross-section area of the copper winding is of $30 \times 21.5 \mathrm{~mm}^{2}$. The coils are placed coaxially around the MAE housing with a separation of $35 \mathrm{~mm}$. Note that this Helmholtz coil is about twice smaller than the one used in test of free bending vibrations (Figure 3) and, in contrast to it, can be regulated using a standard power supply. This follows our

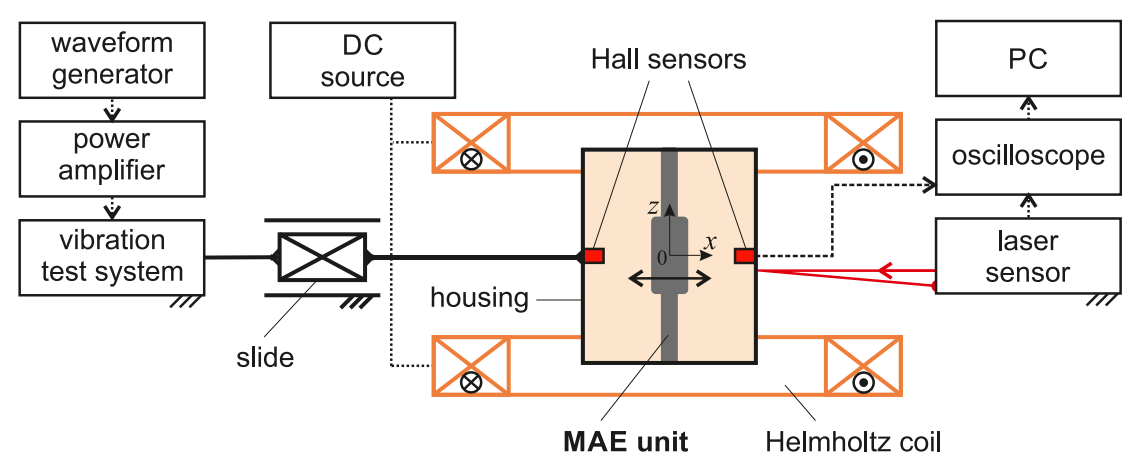

Figure 8: Schematic diagram of the setup used to study forced in-plane bending vibrations of a double-fixed MAE beam in a field of the Helmholtz coil. Reprinted by permission from [50]. 


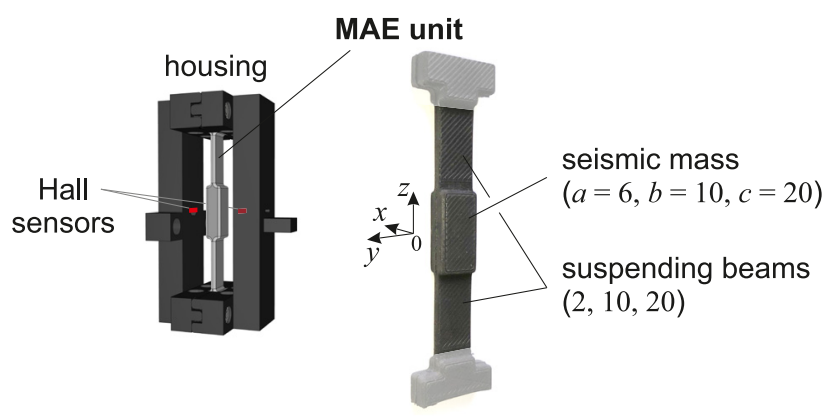

Figure 9: The geometry of the MAE vibrating unit and its double-sided fixation inside the housing. Dimensions are given in $\mathrm{mm}$ with respect to $x, y$ and $z$ axes [50].

attempt to realize a practical design of the intended prototype without laboratory limitations. In the absence of the MAE, the current of $I=2.4 \mathrm{~A}$ in each coil creates an almost uniform magnetic field inside the inner region between the coils. The field is measured using a Hall effect sensor attached to a positioning system and also analyzed using finite element method (ANSYS Maxwell software). The results show that the magnetic induction at the center point is $B_{0}=31.62 \mathrm{mT}$. In the inner centric spherical volume with a diameter of $30 \mathrm{~mm}$, the Helmholtz coil provides a homogeneity of $95 \%$.

To detect changes of the magnetic field caused by the presence of an MAE, two Hall effect sensors (DRV5053, Texas Instruments Inc.) are fixed in the middle of the housing's sides (Figures 7 and 8). In equilibrium, the distance between each sensor and the center point of the MAE beam is $d=12 \mathrm{~mm}$. Both sensors measure the vertical component of magnetic field, when the external field of the same direction is applied. Their sensitivity is $23 \mathrm{mV} / \mathrm{mT}$, that allows measuring fields with magnitudes up to $34.78 \mathrm{mT}$. Such a sensitivity range is sufficient, as in this configuration field distortions on the sides of the MAE are negative, and so the measured field values are smaller than the ones applied [50].

The geometry of the MAE beam is shown in Figure 9. The sample is utilized in the form of a double-fixed elastic beam with a large central part as a seismic mass suspended on two sides. The overall length of the beam without including the fixing parts is $60 \mathrm{~mm}$. The cross-sectional dimensions $2 \mathrm{~mm} \times 10 \mathrm{~mm}$ of two suspending beams are specified by numerical simulations using the finite element method (ANSYS Modal analysis) on condition that the basic eigenfrequency of the unit, being far below the second one, corresponds to the transverse bending. Simulation results show that for the selected sample geometry, its first and second eigenfrequencies corresponding to the free bending and torsion vibrations are 8.24 and $18.65 \mathrm{~Hz}$, respectively. Also, all edges are rounded with a radius of $1 \mathrm{~mm}$, so as to smooth magnetic field distortions at the corners. The MAE beam is made with the use of the same materials and synthesis methods as described in Section 2.1.1. The mass content of the CIP magnetic filler is of 

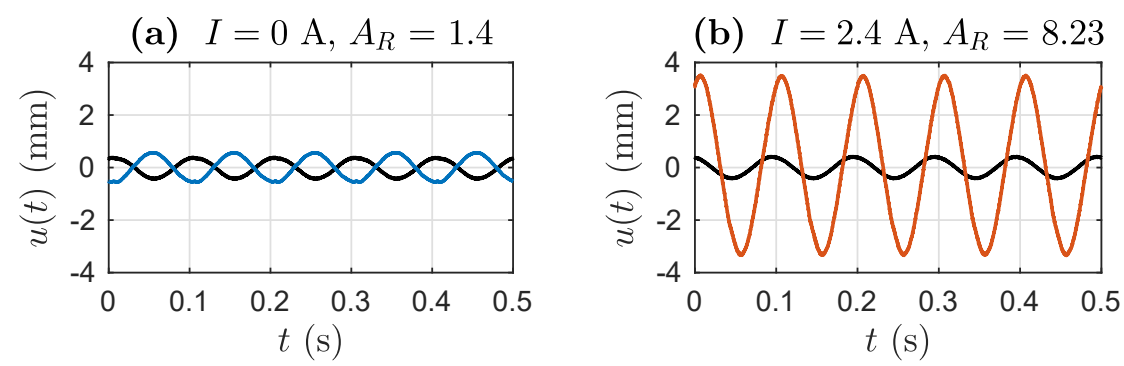

Figure 10: Steady-state displacement $u(t)$ as a time function measured at the middle of the MAE unit for the currents: (a) $I=0 \mathrm{~A}$ (blue line); (b) $I=2.4 \mathrm{~A}$ (orange line). Black line shows the input excitation with $\Omega=10 \mathrm{~Hz}$ and $u_{0}=0.4 \mathrm{~mm}$. Reprinted by permission from [50].

$76.2 \mathrm{wt} \%$, which is nearly $\phi=30 \mathrm{vol} \%$. This is chosen due to the fact that considerable changes of material properties in a moderate magnetic field occur with increasing CIP concentration, as shown in Figure 5.

\subsubsection{Results of forced bending vibrations of the MAE unit}

The steady-state response of the forced, lightly damped in-plane vibrations of the MAE unit is studied experimentally for various excitation frequencies of the housing [50]. Figure 10 shows the displacement recorded by the laser sensor at the middle of the MAE unit for the same applied excitation with $\Omega=10 \mathrm{~Hz}$ in the following two cases: (a) without an external magnetic field; (b) with the field generated by a current of $I=2.4$ A in the Helmholtz coil.

It can be seen that the response vibrations have the same frequency as that of the excitation with a phase shift. Their amplitudes are strongly dependent on the applied field strength. Consider the amplification ratio $A_{R}$ defined as the ratio of the response amplitude to the forcing amplitude, which is of $u_{0}=0.4 \pm 0.02 \mathrm{~mm}$. For the case without magnetic field, the ratio $A_{R}$ is 1.4 and the phase shift is about $170^{\circ}$ corresponding to the high frequency response after the resonance, see Figure 10 (a). In the magnetic field, we have $A_{R}=8.23$ and a phase shift of about $39^{\circ}$, which refers to the frequency response before the resonance, see Figure 10 (b).

Amplification ratio curves as a function of the excitation frequency are presented in Figure 11. It can be seen that these curves have the same qualitative behavior. The application of the magnetic field generated by a current of $I=2.4$ A leads to a shift of the curve's peak to the right. This "tuning" effect is related to the magnetic field-induced change of the vibration characteristics of the MAE unit, primarily its first eigenfrequency of free bending vibrations. The higher the strength of a magnetic field, the stronger shift along the $\Omega$ axis it would cause. As a result, the forced vibration response of the MAE unit can be actively controlled by the strength of an external magnetic field, 


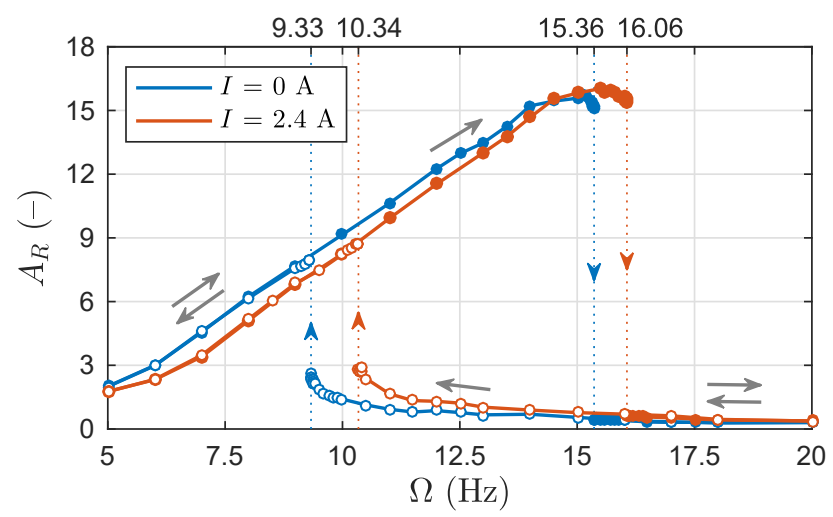

Figure 11: Amplification ratio $A_{R}$ against the excitation frequency $\Omega$ for two currents $I$. Thick and white markers correspond to a step increase and decrease of $\Omega$, respectively. Frequency values of quasidiscontinuous jumps in the response amplitude are ticked on the top axis. Reprinted by permission from [50].

in this case by changing the current supplied to the coil. The "configuration" of the MAE unit can be switched reversibly between the low/high and resonance responses.

A certain asymmetry of the curves of the amplification ratio near the resonance peak shows that there is an element of nonlinearity. Both curves are bent to the right side that is related to the hardening type of behavior. At specific ranges of the excitation, two stable stationary oscillations may exist depending on whether the frequency $\Omega$ increases or decreases stepwise. This bifurcation results in the appearance of quasi-discontinuous jumps in the response amplitude. By increasing the current in the coil, the apparent stiffness of the MAE unit increases, consequently, the hardening effect of nonlinearity decreases. As seen in Figure 11, the frequency width of the bifurcation range changes from 6.03 to $5.72 \mathrm{~Hz}$. As will be seen in the next section, in stronger fields the nonlinear system would tend to approach the linear one [50].

For the case of $\Omega=10 \mathrm{~Hz}$ without applied magnetic field, two stable steady-state responses exist, and Figure 10 (a) corresponds to the one of the lower branch, as the frequency decreases. When $I=2.4 \mathrm{~A}$, there is only one stable response shown in Figure 10 (b).

\subsection{Modeling of nonlinear system behavior under forced vibrations}

A similar nonlinear phenomenon of the resonance hysteresis takes place in mechanics for an oscillating spring-mass system with a cubic stiffness term, which motion is described by Duffing equation [51]. This equation can provide the simplest possible model for the observed forced vibrations of the MAE beam with reasonable qualitative 


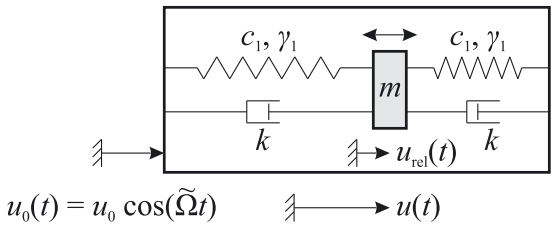

Figure 12: Simplified mechanical model with one degree of freedom for the forced vibrations of an MAE beam.

agreement. It is also applicable to study frequency response of a steel cantilever in the nonuniform magnetic field of two permanent magnets [52].

Assume that due to the symmetry, the central seismic mass $m$ of the MAE beam moves translationally as a solid body in the horizontal direction. As it is connected by the same two suspending beams to a rigid housing, these beams can be modeled as two springs placed on the left and right sides of the mass (Figure 12). The bending stiffness of the suspending beams depends on the Young modulus $E$ of the material, the area moment of inertia $I_{z}$ of the cross-section and length of the beams. Undergoing large deflections, the behavior of the bending stiffness becomes nonlinear. This can be seen as a nonlinear progressive spring force with a cubic stiffness expressed by

$$
F_{c}=c_{1} u_{\text {rel }}+\gamma_{1} u_{\text {rel }}^{3},
$$

where $c_{1}$ and $\gamma_{1}$ are positive spring constants of its linear and cubic terms.

The relative displacement $u_{\text {rel }}(t)$ is the displacement of the mass with respect to the housing, which is in turn subjected to the kinematic excitation $u_{0}(t)=u_{0} \cos (\tilde{\Omega} t)$ in the horizontal direction (Figure 12). Here, $\tilde{\Omega}$ is the angular frequency of excitation. The absolute vibrational motion of the mass is described by the function $u(t)=u_{\text {rel }}(t)+$ $u_{0}(t)$ and can be measured with a laser triangulation sensor.

Considering that vibrations of the suspending beams are lightly damped as shown in Figure 4 (b), the damping force is modeled as being proportional to the relative velocity of the mass with a low viscous damping coefficient $k$. Equation of motion of the mass relative to an inertial reference frame is given by

$$
m\left(\ddot{u}_{\text {rel }}(t)+\ddot{u}_{0}(t)\right)=-2 c_{1} u_{\text {rel }}(t)-2 \gamma_{1} u_{\text {rel }}^{3}(t)-2 k \dot{u}_{\text {rel }}(t) .
$$

This equation represents only a single mode of vibration. The angular eigenfrequency of the translating motion is $\omega_{0}=\sqrt{2 c_{1} / m}$. The damping ratio, cubic stiffness coefficient and the frequency ratio are introduced as dimensionless quantities in the following way:

$$
D=\frac{k}{m \omega_{0}}, \quad \gamma=\frac{2 \gamma_{1} u_{0}^{2}}{m \omega_{0}^{2}}, \quad \eta=\frac{\tilde{\Omega}}{\omega_{0}} .
$$

Equation of motion for the relative displacement can be written in form of the Duffing equation

$$
\ddot{u}_{\text {rel }}(t)+2 D \omega_{0} \dot{u}_{\text {rel }}(t)+\omega_{0}^{2} u_{\text {rel }}(t)+\gamma \frac{\omega_{0}^{2}}{u_{0}^{2}} u_{\text {rel }}^{3}(t)=u_{0} \eta^{2} \omega_{0}^{2} \cos (\tilde{\Omega} t) .
$$


To obtain approximately a steady-state solution of (8), we suppose that it is proportional to the excitation amplitude with an amplification ratio $A_{R \text {,rel }}$ and has an induced phase shift $\varphi_{\text {rel }}$ :

$$
u_{\text {rel }}(t)=u_{0} A_{R, \text { rel }} \cos \left(\tilde{\Omega} t-\varphi_{\text {rel }}\right) .
$$

Substituting this solution form into (8) and using an expression $\cos ^{3}(\alpha)=$ $3 / 4 \cos (\alpha)+1 / 4 \cos (3 \alpha)$, we equate the sine and cosine terms to zero separately:

$$
\begin{array}{ll}
u_{0} \omega_{0}^{2} \cos \left(\tilde{\Omega} t-\varphi_{\text {rel }}\right): & -\eta^{2} A_{R, \text { rel }}+A_{R, \text { rel }}+\frac{3 \gamma}{4} A_{R, \text { rel }}^{3}=\eta^{2} \cos \left(\varphi_{\text {rel }}\right), \\
u_{0} \omega_{0}^{2} \sin \left(\tilde{\Omega} t-\varphi_{\text {rel }}\right): & 2 D \eta A_{R, \text { rel }}=\eta^{2} \sin \left(\varphi_{\text {rel }}\right) .
\end{array}
$$

Note that for an approximate solution, the cosine term of triple angle remains unbalanced.

Denoted by $\eta_{\star}^{2}$, the squared frequency of free translating vibration, dependent on the dimensionless amplitude, is as follows:

$$
\eta_{\star}^{2}=1+\frac{3 y}{4} A_{R, \mathrm{rel}}^{2}
$$

By excluding the phase shift in (9), the dependence of the relative amplification ratio regarding the housing on the frequency ratio is

$$
A_{R, \text { rel }}=\frac{\eta^{2}}{\sqrt{\left(\eta_{\star}^{2}-\eta^{2}\right)^{2}+(2 D \eta)^{2}}} .
$$

For the absolute motion of the mass, we have

$$
u(t)=u_{\text {rel }}(t)+u_{0}(t)=u_{0} A_{R, \text { rel }} \cos \left(\tilde{\Omega} t-\varphi_{\text {rel }}\right)+u_{0} \cos (\tilde{\Omega} t)=u_{0} A_{R} \cos (\tilde{\Omega} t-\varphi),
$$

where the absolute amplification ratio $A_{R}$ is defined by

$$
A_{R}=\sqrt{\frac{\eta_{\star}^{4}+(2 D \eta)^{2}}{\left(\eta_{\star}^{2}-\eta^{2}\right)^{2}+(2 D \eta)^{2}}}
$$

The phase shift between the excitation and absolute motion equals to

$$
\tan (\varphi)=\frac{2 D \eta^{3}}{\eta_{\star}^{4}-\eta_{\star}^{2} \eta^{2}+(2 D \eta)^{2}}
$$

As is seen from (11), the obtained solution of the amplification ratio $A_{R}$ with respect to an inertial frame can be conveniently represented on a graph as a function of the squared frequency ratio $\eta^{2}$. Figure 13 shows the comparison of these theoretical dependencies for the spring-mass model with the experimental results of forced vibrations of the MAE beam (taken from Figure 11). The calculation parameters of the model for both cases without and with magnetic field are the following: 

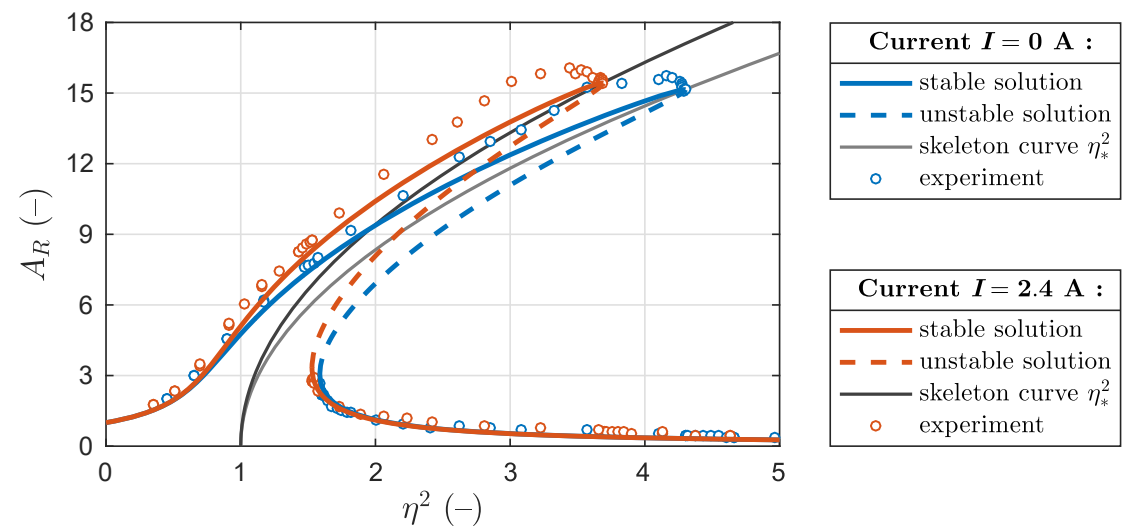

Current $I=2.4 \mathrm{~A}$

stable solution

- - - unstable solution

skeleton curve
experiment

Figure 13: Comparison of the theoretical (lines) and experimental (markers) dependencies of the amplification ratio $A_{R}$ as a function of $\eta^{2}$ for two currents $I$.

$$
\begin{gathered}
I=0 \mathrm{~A}: \quad f_{0}=\frac{\omega_{0}}{2 \pi}=7.41 \mathrm{~Hz}, \quad y=\frac{41.5 \mathrm{~s}^{-2}}{\omega_{0}^{2}}=0.0191, \quad D=0.0685 \\
I=2.4 \mathrm{~A}: \quad f_{0}=\frac{\omega_{0}}{2 \pi}=8.36 \mathrm{~Hz}, \quad \gamma=\frac{41.5 \mathrm{~s}^{-2}}{\omega_{0}^{2}}=0.015, \quad D=0.0623
\end{gathered}
$$

These parameters are chosen so that the theoretical frequency values of the quasidiscontinuous jumps in the response amplitude coincide with the experimental ones. In the range between these frequencies, as Figure 13 shows, Eq. (11) gives three solutions: with a large amplitude (upper branch), with a small amplitude (lower branch) and with an intermediate amplitude. The latter is unstable and can not be implemented practically. One of two stable solutions from the lower or upper branch can be observed depending on whether the excitation frequency increases or decreases monotonously as done in our experiment.

The considered model explains clearly the influence of a magnetic field on the nonlinear effect of MAE vibrations. The range of bifurcation, where both stable solutions exist, depends on the slope of the so-called skeleton curve defined by (10). For $y>0$, it bends to the right characterizing hardening type of behavior. Since the eigenfrequency of free vibration increases in the presence of a magnetic field, the value of $y$ decreases as the square of the frequency, and the damping ratio $D$ is inversely proportional to it, see (7). Therefore, the slope of the skeleton curve relative to the vertical gets smaller, as the current in the Helmholtz coil, i.e. the applied field strength, increases. In the limiting case of strong magnetic field, the nonlinear forced response would tend to approach the behavior of a linear undamped system with a resonance frequency $\eta \simeq 1$ independent of the amplitude. 


\section{Detection of MAE vibrations by magnetic field sensing}

With respect to the intended application of MAE beams as functional sensing elements of an acceleration sensor (Figure 7), the focus of this section is on the unambiguous determination of MAE forced vibrations. Such a vibrating unit placed in an external magnetic field distorts the field distribution over the space around it [50]. By using suitable strategies and ranges of the magnetic field sensing, this information can be used to determine its vibrational motion.

\subsection{Theoretical analysis of MAE magnetostatics}

Consider a coordinate system with the origin at the center point of the MAE vibrating unit in equilibrium, so that the $x-z$ plane is the plane of its vibrations (Figure 8). As in [50], let us simplify the geometry of the unit and take into account only the central seismic mass. It is a rectangular parallelepiped with dimensions of $a=6 \mathrm{~mm}$, $b=10 \mathrm{~mm}, c=20 \mathrm{~mm}$ in $x, y$ and $z$ directions, respectively (Figure 9).

When a uniform magnetic field $B_{0}$ is applied vertically along the $z$ axis, the magnetization $M$ at the center of the parallelepiped points in the same direction. The demagnetizing field is, however, directed opposite to the magnetization. Due to the symmetry, the magnetic field along the $x$ axis has only a vertical component $B_{z}(x)$. Its value inside the parallelepiped is bigger than $B_{0}$. For the region $|x|>a / 2$ outside the body, $B_{z}(x)$ is smaller that $B_{0}$ and tends asymptotically to it with increasing distance.

The strength $H_{z}(x)$ of the static magnetic field around the parallelepiped along the $x$ axis can be calculated using the approximation of a uniform magnetization as follows [53]:

$$
H_{z}(x)=H_{0}+\frac{M}{\pi} \sum_{i=1,2} \arctan \left(\frac{(-1)^{i+1} b x_{i}}{c \sqrt{x_{i}^{2}+\left(b^{2}+c^{2}\right) / 4}}\right)
$$

where $x_{i}=x+(-1)^{i} a / 2, i=1,2$.

This analytical solution shows that $H_{z}(0)=H_{0}-0.26 M / \pi$. For the linear magnetization law, we have $M=\chi H_{z}(0)$, where the magnetic susceptibility $\chi(\phi)=2.72$ of the MAE with a concentration of $\phi=0.3$ is approximated by (2) taking, as before, $\chi_{\mathrm{e}}=79$. Thus, the magnetization of the material at the center is $M=2.23 H_{0}$. It can also be shown that the solution (13) satisfies the Maxwell equations in both regions inside and outside the parallelepiped and the boundary conditions. In particular, on the left and right sides $x= \pm a / 2$, the tangential component $H_{z}$ of the field strength is continuous, while $B_{z}$ undergoes a jump. 


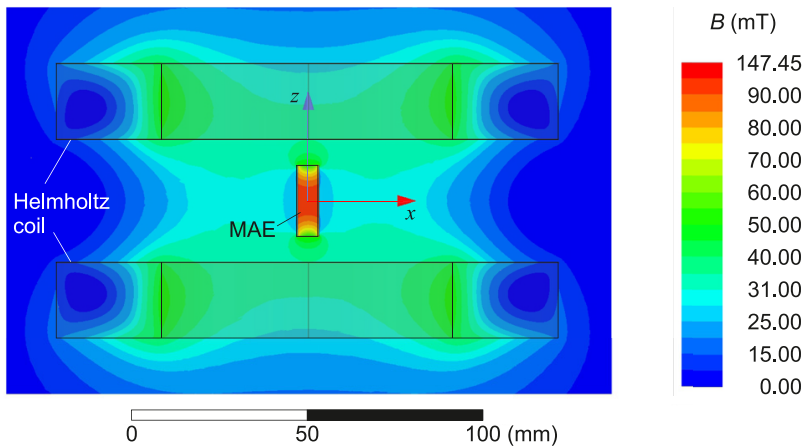

Figure 14: Magnetic induction of an MAE rectangular parallelepiped placed in the middle of the Helmholtz coil as a result of the numerical solution using magnetostatic analysis (ANSYS Maxwell software). Simulation parameters are: excitation current in the coil $I=2.4 \mathrm{~A}$; dimensions of the parallelepiped $a=6 \mathrm{~mm}, b=10 \mathrm{~mm}, c=20 \mathrm{~mm}$; magnetic susceptibility of MAE $X=2.72$.

The numerical solution for the distribution of the magnetic field magnitude around the MAE parallelepiped is obtained using magnetostatic analysis (ANSYS Maxwell software) and shown in Figure 14. The simulation parameters of the Helmholtz coil are the same as for the coil used in experiments, see Section 3.1.1.

When the parallelepiped is displaced from the center point by a distance $u_{\text {rel }}$ along the $x$ axis, it is closer to the right Hall sensor with a distance of $d-u_{\text {rel }}$ between them (Figure 8). So, the distance to the left Hall sensor is $d+u_{\text {rel }}$. In equilibrium, the distance between each sensor and the center point of the MAE beam is $d=12 \mathrm{~mm}$. Both sensors, right and left, are fixed inside the housing so that they measure vertical field components only $B_{z 1}\left(d-u_{\text {rel }}\right)$ and $B_{z 2}\left(d+u_{\text {rel }}\right)$, respectively. As closer the magnetized body is to the sensor, as more field distortion it produces. Therefore, $B_{z 1}<B_{z 2}$ for $u_{\text {rel }}>0$, and vice versa.

The difference of these field values is calculated using (13) as

$$
\begin{aligned}
\Delta B\left(u_{\text {rel }}\right)= & B_{z 1}\left(d-u_{\text {rel }}\right)-B_{z 2}\left(d+u_{\text {rel }}\right)= \\
& =\frac{2.23}{\pi} B_{0} \sum_{i=1,2}\left(\begin{array}{l}
\arctan \left(\frac{(-1)^{i+1} b\left(d_{i}-u_{\text {rel }}\right)}{c \sqrt{\left(d_{i}-u_{\text {rel }}\right)^{2}+\left(b^{2}+c^{2}\right) / 4}}\right) \\
-\arctan \left(\frac{(-1)^{i+1} b\left(d_{i}+u_{\text {rel }}\right)}{c \sqrt{\left(d_{i}+u_{\text {rel }}\right)^{2}+\left(b^{2}+c^{2}\right) / 4}}\right)
\end{array}\right),
\end{aligned}
$$

where $d_{i}=d+(-1)^{i} a / 2, i=1,2$, and $\left|u_{\text {rel }}\right| \leq d-a / 2$.

The series expansion of this dependence at zero contains odd powers only, and the coefficients of the third and higher powers are negligibly small. Hence, the difference of 
the measured field values can be well approximated as a linear function of the displacement $u_{\text {rel }}$ :

$$
\Delta B\left(u_{\text {rel }}\right) \simeq 0.0156 \mathrm{~mm}^{-1} \cdot B_{0} u_{\text {rel }} .
$$

Thus, the considered strategy of the magnetic field measurement at two specific points around the MAE vibrating unit provides a linear relationship between the kinematic variable $u_{\text {rel }}$ and magnetic field magnitude $\Delta B$ measured by the electrical signals of the Hall sensors.

\subsection{Experimental results of magnetic field sensing}

Figure 15 shows the experimental results for the steady-state response of the MAE unit for an excitation frequency of $\Omega=16 \mathrm{~Hz}$ and a current in the coil of $I=2.4 \mathrm{~A}$. In the absence of the magnetic unit, this current generates an almost uniform magnetic field with a magnitude of $B_{0}=31.62 \mathrm{mT}$ at the center point. The horizontal displacement $u(t)$ at the middle point of the MAE unit is recorded by a laser triangulation sensor. The amplitude of this response is $\tilde{u}=6.19 \mathrm{~mm}$. So, the amplification ratio is in this case $A_{R}=15.48$, which means that the resonance response takes place. For the relative motion of the MAE unit about the housing, the amplitude is $\tilde{u}_{\text {rel }}=6.29 \mathrm{~mm}$.

The magnetic field distortion caused by the MAE vibrations is simultaneously measured by two Hall sensors that are fixed on the sides of the housing. As can be seen in Figure 15, the difference $\Delta B(t)$ of the field values of both sensors has the same frequency as that of the excitation. Moreover, its amplitude is of $\Delta \tilde{B}=4.03 \mathrm{mT}$, which is $12.74 \%$ of the applied field $B_{0}=31.62 \mathrm{mT}$. Note that this distortion is slightly bigger than the change of $9.81 \%$ obtained using the theoretical approximation by Eq. (15).
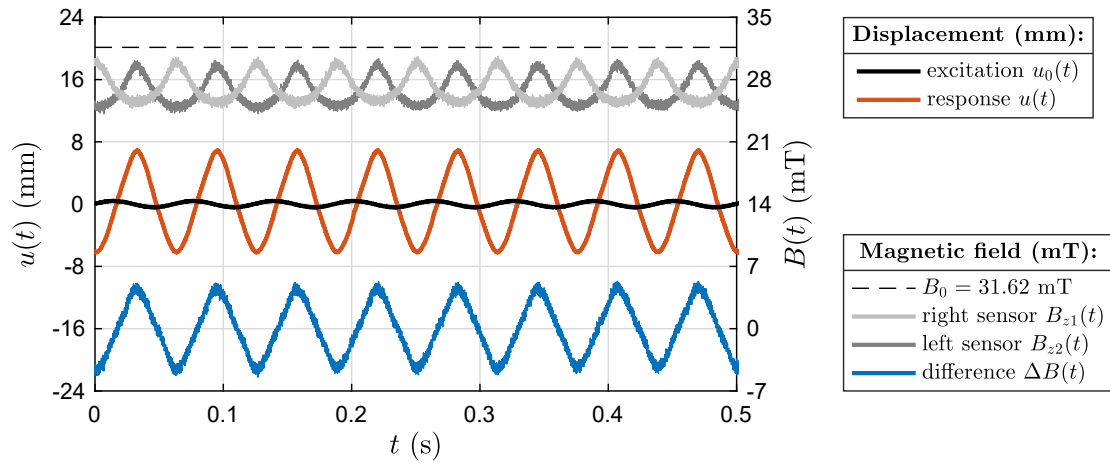

Figure 15: Steady-state response of the MAE vibrating unit recorded by a laser triangulation sensor (orange line) and two Hall sensors (blue line) for an excitation frequency $\Omega=16 \mathrm{~Hz}$, excitation amplitude $u_{0}=0.4 \mathrm{cmm}$ and current of $I=2.4 \mathrm{~A}$. 


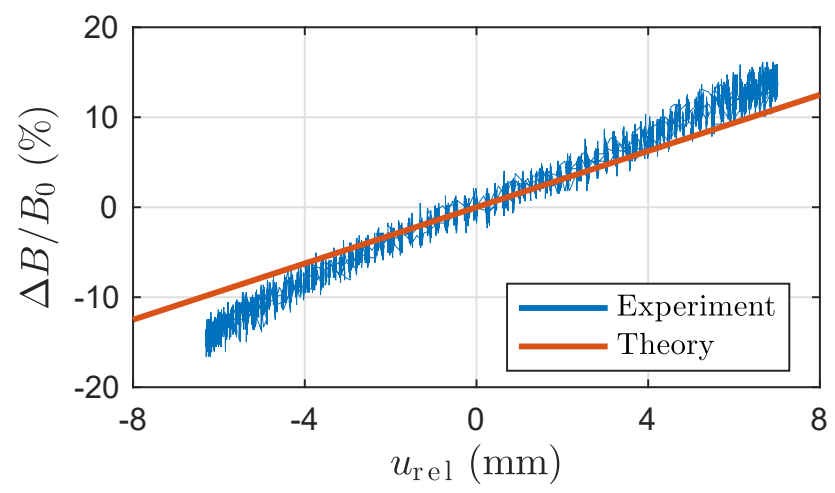

Figure 16: Linear relation of the magnetic field distortion $\Delta B$ on the displacement $u_{\text {rel }}$ of the MAE vibrating unit relative to its housing. Theoretical line is defined by (15).

The experimental results confirm that during the forced bending vibrations of the MAE unit, there is a linear dependence between the relative displacement and the occurring magnetic field distortion (Figure 16). Some disparity of the slopes of the experimental and theoretical lines is caused by the simplifications assumed during the analytical calculations. In particular, Eq. (15) describes only the field change produced by the central seismic part of the MAE unit, and is derived using approximation of its uniform magnetization.

\section{Conclusions}

This work presents the results of experimental and theoretical investigations on MAEs towards an approach of their potential application as vibrating sensor elements with adaptable sensitivity. Starting with the experimental and model-based studies of the free vibrational behavior of cantilevers made of MAEs, it is shown that the first bending eigenfrequency of the cantilevers depends strongly on the strength of an applied uniform magnetic field. The developed theoretical model predicts reliably the dependencies of the vibration characteristics on the geometric configuration and the material parameters of the chosen MAE.

The experimental investigation of the forced vibration response of a double-fixed MAE beam under kinematic excitation confirms the possibility of active magnetic control of the amplitude-frequency characteristics. With change of the uniform field strength, the MAE beam reveals different steady-state responses for the same excitation. The resonance may occur at various ranges of the excitation frequency. Nonlinear dependencies of the amplification ratio on the excitation frequency are obtained for two different applied fields. The model of an oscillating springâ€"mass system with a cubic stiffness term is employed to analyze the influence of the magnetic field on the 
nonlinear phenomenon of the resonance hysteresis. It is shown that the steady-state vibrations of MAE beams can be detected based on the magnetic field distortion. The field difference, which is measured simultaneously on the sides of a vibrating MAE beam, provides a signal with the same frequency as the excitation. The field amplitude is proportional to the amplitude of resulting vibrations.

The presented prototype of the MAE-based vibrating unit with the field-controlled "configuration" can be implemented for realization of acceleration sensor systems with adaptable sensitivity. Such an MAE unit transforms mechanical stimuli of its housing into bending vibrations that, in turn, could be converted in a facile way into electromagnetic signals of Hall sensors for measuring and analyzing. Due to the exceptional field-dependent properties, the MAE vibrating unit can be used both in a resonance mode or in a regime of low/high responses. The ongoing research on MAEs is oriented to the use of other geometrical forms along with beams, e.g. two-dimensional structures such as membranes.

Acknowledgment: This research is funded by the Deutsche Forschungsgemeinschaft (DFG) in the framework of the priority program SPP 1681 under the projects BE 6553/1-1 and ZI 540-17/3. The support of the research association PAK 907 between the DFG and the Russian Foundation for Basic Research (RFBR) is also gratefully acknowledged (project 19-52-12045). We thank our scientific colleagues and their teams in the research association PAK 907: D. Borin (Dresden), G. Stepanov (Moscow), S. Kantorovich and A. Zubarev (Ekaterinburg). We thank all working groups of SPP 1681 for the great scientific work they are performing in the respective subprojects. We thank specially S. Odenbach (Dresden) for his efforts in the coordination of both research programs.

Author contribution: All the authors have accepted responsibility for the entire content of this submitted manuscript and approved submission.

Research funding: None declared.

Conflict of interest statement: The authors declare no conflicts of interest regarding this article.

\section{References}

1. Carlson JD, Jolly MR. MR fluid, foam and elastomer devices. Mechatronics 2000;10:555-69.

2. Bossis G, Volkova O, Lacis S, Meunier A. Magnetorheology: fluids, structures and rheology. In: Odenbach S, editor. Ferrofluids: Magnetically Controllable Fluids and Their Applications, SpringerVerlag Berlin Heidelberg: Lect Notes Phys, LNP; 2002, vol 594, pp. 202-30.

3. Jolly MR, Carlson JD, Munoz BC, Bullions TA. The magnetoviscoelastic effect of elastomer composites consisting of ferrous particles embedded in a polymer matrix. J Intell Mater Syst Struct 1996;7:613-22.

4. Kallio M. The elastic and damping properties of magnetorheological elastomers, Espoo: VVT Publications; 2005, vol 565:146 p. 
5. Varga Z, Filipcsei G, Zrínyi M. Magnetic field sensitive functional elastomers with tuneable elastic modulus. Polymer 2006;47:227-33.

6. Lee D, Lee M, Jung N, Yun M, Lee J, Thundat T, et al. Modulus-tunable magnetorheological elastomer microcantilevers. Smart Mater Struct 2014;23: 055017.

7. Volkova TI, Böhm V, Kaufhold T, Popp J, Becker F, DYu B, et al. Motion behaviour of magnetosensitive elastomers controlled by an external magnetic field for sensor applications. J Magn Magn Mater 2017;431:262-5.

8. Koo JH, Dawson A, Jung HJ. Characterization of actuation properties of magnetorheological elastomers with embedded hard magnetic particles. J Intell Mater Syst Struct 2010;23:1049-54.

9. Kramarenko EY, Chertovich AV, Stepanov GV, Semisalova AS, Makarova LA, Perov NS, et al. Magnetic and viscoelastic response of elastomers with hard magnetic filler. Smart Mater Struct 2015;24: 035002.

10. Böse H, Hesler A, Monkman G. Magnetorheological composite materials comprising hard magnetic particles, method for the production thereof and use thereof. United States: Google Patent DE Patent 102007028663 A1; 2007.

11. Stepanov GV, Borin DY, Storozhenko PA. Rotation of magnetic particles inside the polymer matrix of magnetoactive elastomers with a hard magnetic filler. J Magn Magn Mater 2017;431:138-40.

12. Borin DY, Stepanov GV. Oscillation measurements on magnetoactive elastomers with complex composition. J Optoelectron Adv Mater 2013;15:249-53.

13. Becker TI, Stolbov OV, Borin DY, Zimmermann K, Raikher YuL. Basic magnetic properties of magnetoactive elastomers of mixed content. Smart Mater Struct 2020;29: 075034.

14. Borin D, Stepanov G, Dohmen E. Hybrid magnetoactive elastomer with a soft matrix and mixed powder. Arch Appl Mech 2019;89:105-17.

15. Remmer H, Roeben E, Schmidt AM, Schilling M, Ludwig F. Dynamics of magnetic nanoparticles in viscoelastic media. J Magn Magn Mater 2017;427:331-5.

16. Schümann M, Borin D, Huang S, Auernhammer GK, Müller R, Odenbach S. A characterisation of the magnetically induced movement of NdFeB-particles in mag-netorheological elastomers. Smart Mater Struct 2017;26: 095018.

17. Linke JM, Borin DY, Odenbach S. First-order reversal curve analysis of magnetoactive elastomers. RSC Adv 2016;6:100407-16.

18. Vaganov MV, Linke J, Odenbach S, Raikher YuL. Model FORC diagrams for hybrid magnetic elastomers. J Magn Magn Mater 2017;431:130-3.

19. Odenbach S. Microstructure and rheology of magnetic hybrid materials. Arch Appl Mech 2016;86: 269-79.

20. Biller AM, Stolbov OV, Raikher YuL. Mesoscopic magnetomechanical hysteresis in a magnetorheological elastomer. Phys Rev E 2015;92: 023202.

21. Tarama M, Cremer P, Borin DY, Odenbach S, Löwen H, Menzel AM. Tunable dynamic response of magnetic gels: impact of structural properties and magnetic fields. Phys Rev E 2014;90: 042311.

22. Goh S, Menzel AM, Löwen H. Dynamics in a one-dimensional ferrogel model: relaxation, pairing, shock-wave propagation. Phys Chem Chem Phys 2018;20:15037.

23. Weeber R, Kreiss P, Holm C. Studying the field-controlled change of shape and elasticity of magnetic gels using particle-based simulations. Arch Appl Mech 2019;89:3-16.

24. Gebhart P, Wallmersperger T. A general framework for the modeling of porous ferrogels at finite strains. J Mech Phys Solid 2019;122:69-83.

25. Metsch P, Kalina KA, Brummund J, Kästner M. Two- and three-dimensional modeling approaches in magneto-mechanics: a quantitative comparison. Arch Appl Mech 2019;89:47-62.

26. Raikher YuL, Stolbov OV. Numerical modeling of large field-induced strains in ferroelastic bodies: continuum approach. J Phys Condens Matter 2008;20:204126. 
27. Biller AM, Stolbov OV, Raikher YuL. Modeling of particle interactions in magnetorheological elastomers. J Appl Phys 2014;116. https://doi.org/10.1063/1.4895980. 114904.

28. Kozlowska J, Boczkowska A, Czulak A, Przybyszewski B, Holeczek K, Stanik R, et al. Novel MRE/CFRP sandwich structures for adaptive vibration control. Smart Mater Struct 2016; 25: 035025.

29. Yang J, Sun SS, Du H, Li WH, Alici G, Deng HX. A novel magneto-rheological elastomer isolator with negative changing stiffness for vibration reduction. Smart Mater Struct 2014;23. https://doi.org/ 10.1088/0964-1726/23/10/105023. 105023.

30. Kim MS, Yang KM, Lee SH, Yoon JH, Jeong UC, Yang IH, Oh JE. Variable differential mount apparatus using magnetorheological elastomer. U.S. Patent 8,844,914 B2; 2014.

31. Thorsteinsson F, Gudmundsson I, Lecomte C. Prosthetic and orthotic devices having magnetorheological elastomer spring with controllable stiffness. U.S. Patent 9,078,734 B2; 2015.

32. Oh J-S, Choi S-B. State of the art of medical devices featuring smart electro-rheological and magneto-rheological fluids. J King Saud Univ Sci 2017;29:390-400.

33. El Wahed AK, Balkhoyor LB. The performance of a smart ball-and-socket actuator applied to upper limb rehabilitation. J Intell Mater Syst Struct 2018;29:2811-22.

34. Zimmermann K, Naletova VA, Zeidis I, Turkov VA, Kolev E, Lukashevich MV, et al. A deformable magnetizable worm in a magnetic field - A prototype of a mobile crawling robot. J Magn Magn Mater 2007;311:450-3.

35. Zimmermann K, Zeidis I, Böhm V, Kaufhold T, Volkova T, Krautz M, et al. Mechanics of actuators based on magnetic hybrid materials with application for robotics, fluid control and sensor technology. IFToMM Probl Mech 2014;57:23-41.

36. Lum GZ, Ye Z, Dong X, Marvi X, Erin O, Hu W, et al. Shape-programmable magnetic soft matter. Proc Natl Acad Sci Unit States Am 2016;113:E6007-15.

37. Gao W, Wang L, Wang X, Liu H. Magnetic driving flowerlike soft platform: biomimetic fabrication and external regulation. ACS Appl Mater Interfaces 2016;8:14182-9.

38. Becker TI, Böhm V, Chavez Vega J, Odenbach S, Raikher YL, Zimmermann K. Magnetic-fieldcontrolled mechanical behavior of magneto-sensitive elastomers in applications for actuator and sensor systems. Arch Appl Mech 2019;89:133-52.

39. Zimmermann K, Chavez Vega J, Becker TI, Witte H, Schilling C, Köhring S, et al. An approach to a form-adaptive compliant gripper element based on magneto-sensitive elastomers with a bioinspired sensorized surface. Int Sci J Probl Mech 2019;75:23-38.

40. Schmauch MM, Mishra SM, Evans BA, Velev OD, Tracy JB. Chained iron microparticles for directionally controlled actuation of soft robots. ACS Appl Mater Interfaces 2017;9:11895-901.

41. Becker TI, Raikher YuL, Stolbov OV, Böhm V, Zimmermann K. Dynamic properties of magnetosensitive elastomer cantilevers as adaptive sensor elements. Smart Mater Struct 2017;26: 095035.

42. Ramanujan RV, Lao LL. The mechanical behavior of smart magnet-hydrogel composites. Smart Mater Struct 2006;15:952-6.

43. Abramchuk S, Kramarenko E, Grishin D, Stepanov G, Nikitin L, Filipcsei G, et al. Novel highly elastic magnetic materials for dampers and seals: Part II. Material behavior in a magnetic field. Polym Adv Technol 2007;18:513-8.

44. Svetlitsky VA. Dynamics of Rods. Berlin Heidelberg: Springer-Verlag; 2005:448 p.

45. Magnus K, Popp K, Sextro W. Schwingungen. Wiesbaden: Springer Vieweg; 2013:298 p.

46. Kneller E. Ferromagnetismus. Berlin: Springer; 1962:816 p.

47. Osborn JA. Demagnetizing factors of the general ellipsoid. Phys Rev 1945;67:351-7.

48. Zakri T, Laurent JP, Vauclin M. Theoretical evidence for 'Lichtenecker's mixture formulae' based on the effective medium theory. J Phys D Appl Phys 1998;31:1589-94.

49. Bozorth RM. Ferromagnetism. Wiley-IEEE Press; 1993:992 p. 
50. Becker TI, Böhm V, Schale F, Zimmermann K. Vibrating sensor unit made of a magnetoactive elastomer with field-adjustable characteristics. J Magn Magn Mater 2020;498:166196. https://doi.org/10.1016/j.jmmm.2019.166196.

51. Guckenheimer J, Holmes P. Nonlinear oscillations, dynamical systems, and bifurcations of vector fields. Appl Math Sci. 1983;42:462 p.

52. Moon FC, Holmes PJ. A magnetoelastic strange attractor. J Sound Vib 1979;65:275-96.

53. Pshenichnikov AF. Magnetic field in the vicinity of a single magnet. Magnetohydrodynamics 1993; 29:37-40. 